\title{
COMMENTARY
}

\section{'Cooling and cathing' the post-resuscitated}

\author{
Karl B Kern* \\ See related research by Cronier et al., http://ccforum.com/content/15/3/R122
}

\begin{abstract}
Cronier and co-workers provide additional evidence that routine use of mild therapeutic hypothermia combined with emergent coronary angiography and percutaneous intervention results in excellent survival with intact neurological function for post-resuscitation patients with ventricular fibrillation.
\end{abstract}

Determining priorities for optimal post-resuscitation care is challenging. Cronier and colleagues [1] share their experience with combining routine coronary angiography with induction of mild hypothermia for patients successfully resuscitated from cardiac arrest. Most recognize the value of therapeutic hypothermia in the treatment of the post-cardiac arrest patient $[2,3]$. But other suggested therapies have limited efficacy data, leaving both the treating physician and medical institutions uncertain about what else to do and how to do it. In a recent issue of Critical Care, Cronier and colleagues share their experience in performing routine coronary angiography and percutaneous intervention (PCI) after successful resuscitation from out-of-hospital cardiac arrest due to ventricular fibrillation [1]. They achieved a 54\% survival to discharge rate with $90 \%$ of survivors having favorable neurological status in 111 patients who had coronary angiography with or without PCI after being resuscitated from out-of-hospital ventricular fibrillation. About half of all patients had PCI for an angiographically identified culprit lesion suspected of causing their cardiac arrest. Subdividing their study population by age, they found no significant differences in outcome among those less than 75 years of age. The number of patients older than 75 years was too small for meaningful comparison.

Mild therapeutic hypothermia was part of their postresuscitation therapeutic bundle of care and was performed in $86 \%$ of all patients. In the remaining

*Correspondence: kernk@email.arizona.edu

Section of Cardiology, University of Arizona College of Medicine, University

Medical Center, 1501 N. Campbell Avenue, Tucson, AZ 85724, USA patients, therapeutic hypothermia was not performed because of severe hemodynamic instability or moribund status. Target temperature $\left(32\right.$ to $34^{\circ} \mathrm{C}$ ) was reached in $81 \%$ of patients. These proportions of patients receiving hypothermia and reaching temperature goal compare favorably with other reports [4].

Though all patients were considered for coronary angiography regardless of their electrocardiographic findings, those considered 'hemodynamically unstable (blood pressure $<90 \mathrm{mmHg}$ with or without epinephrine for 30 minutes or more) were not. These unstable patients might be those most in need of urgent coronary angiography post-cardiac arrest. The 'sickest' patients are often the ones with the most to gain by an aggressive approach. Unfortunately, by their exclusion no data were obtained in this 'unstable' group. However, their data evaluating the use of early coronary angiography with or without PCI in post-cardiac arrest patients regardless of their post-resuscitation electrocardiogram (ECG) showing ST elevation or not is very helpful. Many now believe that all ST elevation myocardial infarction patients, whether they have suffered cardiac arrest or not, should undergo timely emergent coronary angiography ( $<90$ minutes 'door-toballoon' time). This includes ST-elevation myocardial infarction (STEMI) patients still comatose post-cardiac arrest. More controversial is whether post-cardiac arrest patients without ST elevation on their ECG should have emergent coronary angiography in the like manner. Previous data, all case series (non-randomized), at least suggest that a substantial proportion of these postresuscitation patients without ST elevation also benefit from emergent cardiac catheterization and PCI [5-8].

In this current report from Cronier and colleagues, 50 patients had ST elevation and 61 patients did not. Survival was similar for both groups (54\% versus 56\%). More patients with ST elevation underwent emergency coronary angiography (94\% versus $72 \%$ ) and more received PCI (79\% versus 20\%). Though no angiographic details are provided, they did find that one out of five resuscitated patients without ST elevation had a culprit lesion requiring acute $\mathrm{PCI}$. This is similar to other reports suggesting that 20 to $30 \%$ of such patients have treatable culprit lesions found at acute coronary angiography [5-8]. The key question is whether one out of five $(20 \%)$ is 
enough to justify routine coronary angiography in all post-resuscitated patients without ST elevation on their ECG? Many think so. Dumas and colleagues [7] recently reported similarly that survival did not correlate with the presence or absence of ST elevation on the post-resuscitation ECG, but rather on whether PCI was performed successfully or not [7]. In patients with no obvious extracardiac etiology and no ST elevation on ECG, routine coronary angiography disclosed coronary heart disease in $58 \%$ of patients without ST elevation and PCI was performed in $26 \%$ of them. They recommended 'the use of immediate coronary angiography in patients with outof-hospital cardiac arrest with no obvious noncardiac cause regardless of ECG pattern' [7].

In the present study, routine coronary angiography after resuscitation whatever the ECG pattern (that is, ST elevation or not), allowed acute detection and treatment of coronary lesions felt to be responsible for the cardiac arrest in $49 \%$ of their 111 studied patients [1]. Importantly, PCI was independently associated with survival in their patient population with stabilized hemodynamics [1].

This report again verifies that those who survive with aggressive post-resuscitation care are very much neurologically intact. The persistent fear that such an approach may only save 'hearts' while 'brains' will suffer, leaving the individual markedly dependent on others, is simply not true. Ninety percent of their survivors had favorable neurological function at discharge.

Aggressive post-resuscitation treatment, including mild therapeutic hypothermia combined with routine coronary angiography and potential PCI, irrespective of the presence of ST elevation or not, is the best opportunity for increasing neurologically intact survival. Let's give these patients their best chance for good long-term neurologically intact survival. That best chance includes 'cooling and cathing' them as soon as they arrive at the hospital.

\section{Abbreviations}

ECG, electrocardiogram; PCl, percutaneous intervention.

Published: 8 August 2011

\section{References}

1. Cronier P, Vignon P, Bouferrache K, Aegerter P, Charron C, Templier F, Castro S, Mahmoud RE, Lory C, Pichon N, Dubourg O, Vieillard-Brown A: Impact of routine percutaneous coronary intervention after out-of-hospital cardiac arrest due to ventricular fibrillation. Crit Care 2011, 15:R122.

2. The HACA Study Group: Mild hypothermia to improve the neurologic outcome after cardiac arrest. N Engl J Med 2002, 346:549-556.

3. Bernard S, Gray TW, Buist MD, Jones BM, Silvester W, Gutteridge G, Smith K: Treatment of comatose survivors of out-of-hospital cardiac arrest with induced hypothermia. N Engl J Med 2002, 346:557-563.

4. Sunde K, Pytte M, Jacobsen D, Mangschau A, Jensen LP, Smedsrud C, Draegni T, Steen PA: Implementation of a standardized treatment protocol for post resuscitation care after out-of-hospital cardiac arrest. Resucitation 2007, 73:29-39.

5. Anyfantakis ZA, Baron G, Aubry P, Himbert D, Fledman LJ, Juliard J-M, Burnod A, Cokkinos DV, Steg PG: Acute coronary angiographic findings in survivors of out-of-hospital cardiac arrest. Am Heart J 2009, 157:312-318.

6. Kern KB, Rahman O: Emergent percutaneous coronary intervention for resuscitated victims of out-of-hospital cardiac arrest. Cath Cardiovasc Interv 2010, 75:616-624.

7. Dumas F, Cariou A, Manzo-Silberman S, Grimaldi D, Vivien B, Rosencher J, Empana J-P, Carli P, Mira J-P, Jouven X, Spaulding C: Immediate percutaneous coronary intervention is associated with better survival after out-ofhospital cardiac arrest. Circ Cardiovasc Interv 2010, 3:200-207.

8. Radsel P, Knafelj R, Kocjancic S, Noc M: Angiographic characteristics of coronary disease and postresuscitation electrocardiograms in patients with aborted cardiac arrest outside a hospital. Am J Cardio/ 2011 [Epub ahead of print].

doi:10.1186/cc10299

Cite this article as: Kern KB: 'Cooling and cathing' the post-resuscitated. Critical Care 2011, 15:178 